\title{
Renormalization of the brain connectome: Duality of particle and wave
}

\author{
S. Petkoski ${ }^{1}$, V.K. Jirsa ${ }^{1} * \dagger$ \\ ${ }^{1}$ Aix-Marseille Univ, Inserm, INS, Institut de Neurosciences des Systèmes, 13005 Marseille, \\ France. \\ *Correspondence to: spase.petkoski@univ-amu.fr \& viktor.jirsa@univ-amu.fr \\ $\dagger$ Both authors equally contributed to the work.
}

\begin{abstract}
:
Networks in neuroscience determine how brain function unfolds. Perturbations of the network lead to psychiatric disorders and brain disease. Brain networks are characterized by their connectomes, which comprise the totality of all connections, and are commonly described by graph theory. This approach is deeply rooted in a particle view of information processing, based on the quantification of informational bits such as firing rates. Oscillations and brain rhythms demand, however, a wave perspective of information processing based on synchronization. We extend traditional graph theory to a dual particle-wave-perspective, integrate time delays due to finite transmission speeds and derive a renormalization of the connectome. When applied to the data base of the Human Connectome project, we explain the emergence of frequency-specific network cores including the visual and default mode networks. These findings are robust across human subjects $(\mathrm{N}=100)$ and are a fundamental network property within the wave picture. The renormalized connectome comprises the particle view in the limit of infinite transmission speeds and opens the applicability of graph theory to a wide range of novel network phenomena, including physiological and pathological brain rhythms.
\end{abstract}

One Sentence Summary: Spatiotemporal and topological network properties are unified within a novel common framework, the renormalized connectome, that explains the organization of fundamental frequency-specific network cores.

\section{Main Text:}

Introduction. Network theory significantly advanced our understanding of complex processes in nature, ranging from gene regulation of protein interactions (1), coordination of brain activity (2) to social networks (3). Connectivity is the dominant concept that shapes the capacity of a network to transmit information (4) and is described by its topological and statistical properties (5). In neuroscience, network theory has been applied on several scales, including microscopic neocortical microcircuitry (6), macroscopic structural connectivity (7), and networks of coordinated brain activity, so-called functional connectivity (8). A connectome is the comprehensive map of neural connections in the brain and maybe thought of as its wiring diagram (9). Models of brain networks based on human connectomes (10) have demonstrated individual predictive power in resting state paradigms (11), cognitive tasks (12) and brain disease such as epilepsy $(13,14)$. In all these network applications, there remains a deeply rooted understanding of signal transmission in the sense of Shannon (15), in which bits of information are transmitted between network nodes. Useful insights about information processing are 
obtained by descriptors such as information capacity (15), transfer entropy (16), mutual information (17) or graph theoretical quantities such as centrality, modularity and hubs (18). In physics, such a picture corresponds to the particle view of classical mechanics, in which the dynamics of particles are a powerful means of describing extended processes and interactions. But, as the particle picture is complemented by a wave picture in physics, similarly in neuroscience there are oscillatory processes deeply implicated in healthy and pathological brain activations, such as cognitive functions (19) or aberrant discharges in epilepsy (20). Such rhythms and waves (in case of spatially extended oscillations) do not have a fundamental description within the particle view of information processing. They are dual to the current view of connectomics in neuroscience, which supports only the particle picture. While the particle view describes network processes by activations, the wave picture imposes a language in terms of synchronization. The importance of the particle-wave duality in neuroscience becomes evident when considering signal transmission delays, present in large scale brain networks and ranging on the order of 10-200 ms (21). As physiological rhythms in the brain are in the same range (22), shifts in arrival times due to delays have minimal to no effects in the particle view, but have consequential frequency dependent effects in the wave view, where a synchronized pair of oscillators may switch from full synchrony to anti-synchrony only due to changes in time delays. As such, frequency and time delays become inseparable properties of the network and together with the connectome determine the information processing capacity of a network (23-25). This also confirms previous computational studies about the effects of the space-time structure of the brain in its emergent dynamics $(26,27)$. Current state of the art network neuroscience does not reach beyond the topological aspects in describing the connectivity of the brain $(2,28)$, thus limits itself to the particle view only and omits space and physical distances between the interacting units (29). In the rare cases when actual lengths of the links are studied (30), it is still done in static manner, without consideration for the impact of the delays on the emerging dynamics, thus critically demanding the extension of network neuroscience to the wave picture.

Particle versus wave representation for network dynamics. The particle-wave dichotomy appears in networks dynamics due to the finite transmission velocities for the signals between spatially distributed network nodes. Within the particle view, the general network dynamics is governed by

$$
\dot{r}_{i}=g_{1}\left(r_{i}\right)+\sum_{j} w_{i j} g_{2}\left(r_{j}\left(t-\tau_{i j}\right)\right) .
$$

Here $r_{i}$ is any scalar variable such as the firing rate, $w_{i j}$ are the weights and $\tau_{i j}$ are the timedelays of the links, and $g_{1}$ and $g_{2}$ are functions for the intrinsic and coupling respectively. This type of particle-like interactions are frequency-independent and here the information and the activity always flow along the links with the strongest coupling weight.

On the other hand, networks of oscillators are often used to conceptualize and to study dynamical systems for which the local activity is multidimensional and nonlinear (31). They have been conceptualized to be responsible for the communication in the brain through coherence (32) and synchronization $(33,34)$. Dynamics of the oscillatory network nodes are governed by

$$
\dot{\boldsymbol{x}}_{i}=F\left(x_{i}\right)+\sum_{j} w_{i j} h\left(\boldsymbol{x}_{j}\left(t-\tau_{i j}\right)\right),
$$

where $\boldsymbol{x}_{\boldsymbol{i}}$ is a vector of states, $F$ is a non-linear function, and $h$ is coupling function. When delays are negligible compared to the time-scale of the system, the interactions are still governed only by the weights, as in the particle case. Due to the better tractability and the readily applicable 
tools from the graph theory, this has been the default network representation in neuroscience. However, when the time-delays are comparable to the time-scale of the intrinsic oscillations, they need to be included in the analysis of network dynamics. As a consequence, this system can only be solved numerically, as there is no alternative graph theoretical approach that integrates the topology and the impact of the time-delays on the oscillations at a given frequency. Hence the systematic effect of the time-delays on the emergent dynamics is concealed and even the full representation of network interactions in Eq. (2), with separated $w_{i j}$ and $\tau_{i j}$ cannot adequately identify the skeleton of the wave synergy.

For weak couplings, dynamics of the system in Eq. (2) is captured by phase models. The simplest and hence most elaborate is the Kuramoto model (35). It is widely utilized for describing emergent phenomena in complex systems (36), with a structure often represented via complex networks (37). For synchronization at frequency $\Omega$, the model reads (38)

$$
\dot{\theta}_{i}=\omega_{i}-\sum_{j} w_{i j} \sin \left(\theta_{i}-\theta_{j}\left(t-\tau_{i j}\right)\right)=\omega_{i}-\sum_{j} w_{i j} \sin \left(\theta_{i}-\theta_{j}+\Omega \tau_{i j}\right),
$$

where $\theta_{i}$ denotes the phase of the $i$-th node oscillating with a natural frequency $\omega_{i}$. From here, the phase difference between each pair of Kuramoto oscillators can be separated from the effect of the rest of the network (see the Supplementary Material), leading to solutions given as

$$
\Delta \varphi_{i j}=\sin ^{-1} \frac{\Delta \omega_{i j}-I_{i j}}{w_{i j} \cos \Omega \tau_{i j}}=\frac{\Delta \omega_{i j}-I_{i j}}{w_{i j}^{(f)}} .
$$

Here $\Delta \omega_{i j}=\omega_{i}-\omega_{j}$ is the frequency mismatch between the two oscillators, $w_{i j}^{(f)}=$ $w_{i j} \cos \Omega \tau_{i j}$ is the impact of the direct link between the pair of oscillators, and $I_{i j}$ contains the influence of all the other links towards these two nodes. For particle-like communication in the limit $\Omega \tau_{i j} \rightarrow 0$ the cosine term and $I_{i j}$ vanish, simplifying Eq. (4) to $\Delta \varphi_{i j}=\sin ^{-1}\left(\Delta \omega_{i j} / w_{i j}\right)$. This makes coupling weights the only factor shaping the network dynamics, consistent with the case of no delays.

Graph theoretical metrics for wave interactions. To go beyond the static representation of networks, the motivation for network wave coupling $w_{i j}^{(f)}$ comes naturally from Eq. (4). It modulates the weight with the impact of the time-delays at each frequency, hence unifying spatio-temporal aspects of each link. More importantly the term $I_{i j}$ in Eq. (4) vanishes for many symmetric cases (see the Supplementary Material), rendering wave-couplings the sole network determinant for synchronization.

As illustrated in Fig. 1 (A top), two delay-coupled Kuramoto oscillators can synchronize either in-phase or anti-phase $(24,31)$, depending on the sign of $\cos \Omega \tau$. The former is stable for positive wave couplings, the latter for negative. The same is true for networks $(23,24)$ and not limited to phase oscillators (25). Time-delays can be perfectly spatially distributed to cause maximum synchronization at a given frequency as shown in the middle plot of panel (A). A more realistic scenario is when the time-delays are distributed in such a manner that some links decrease the network synchronization (if $w_{i j}^{(f)}>0$ for anti-phase nodes, or if $w_{i j}^{(f)}<0$ otherwise) for any phase arrangement. One possibility here is the oscillators rearranging their phases to minimize this disturbance Fig. 1 (A bottom). 
(A)
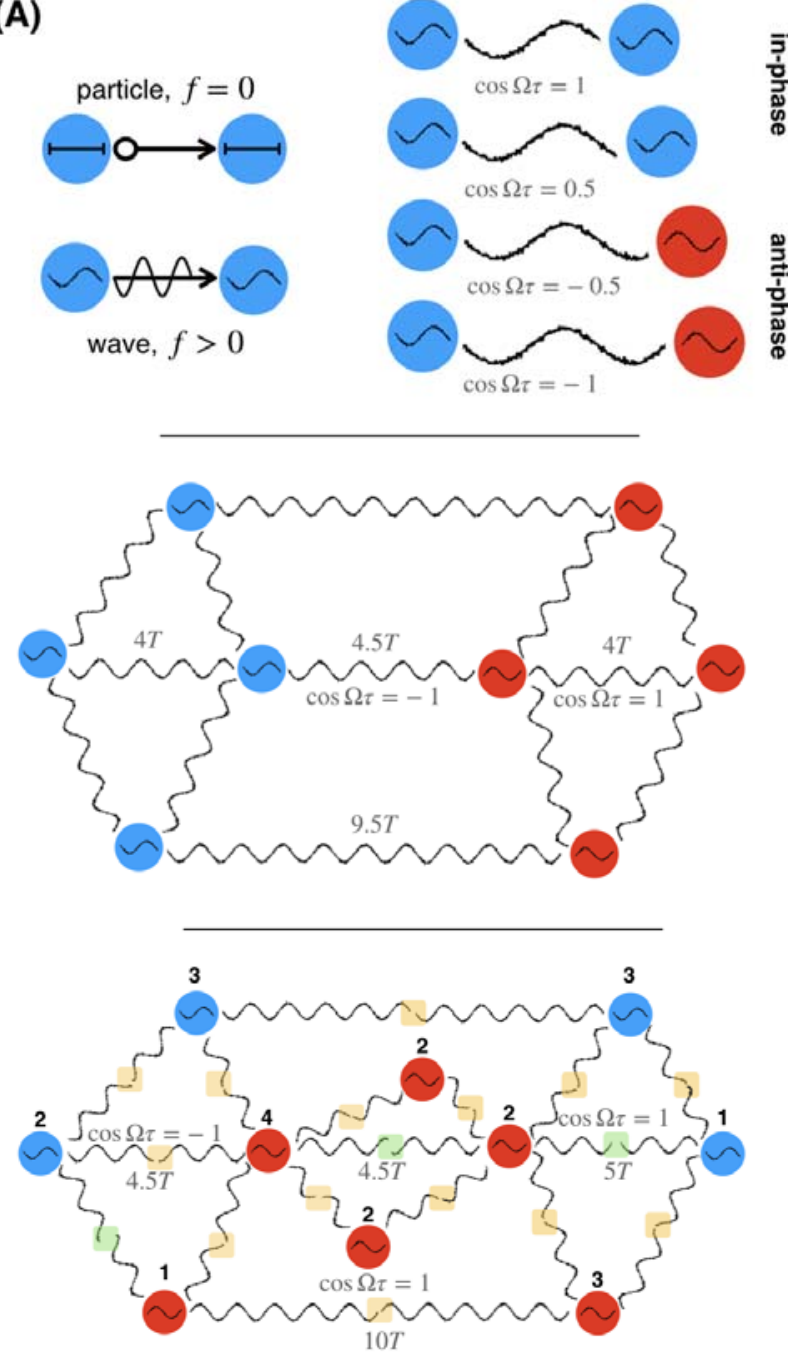

(B)
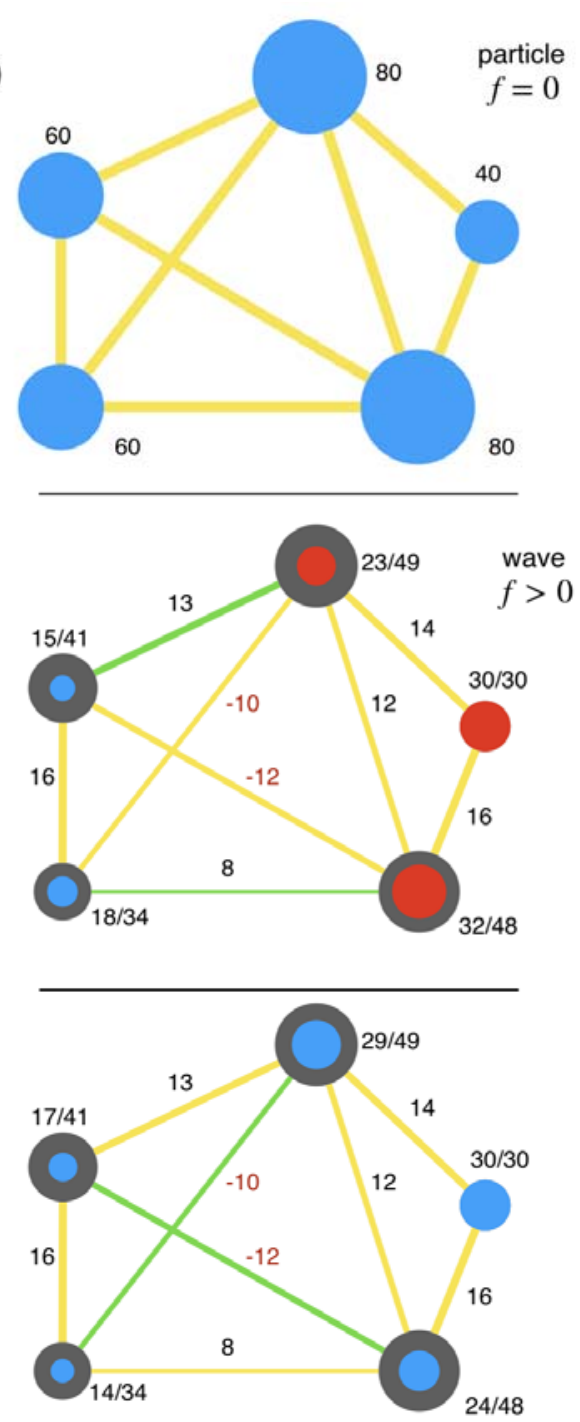

Fig. 1. Spatio-temporal network organization and the wave coupling weights for synchronized networks. (A) Particle and wave type communication over a link with delay (top left). In-and antiphase synchronization for fixed frequency and different time-delays for 2 oscillators (top right), and for networks with a resonant (middle) and non-resonant (bottom) spatio-temporal alignment of the oscillators. Nodes with same color are in-phase with each other, and anti-phase otherwise. Time-delays are illustrated in number of periods of the frequency of synchronization and the renormalization factor is shown for some of the links. (B) Wave coupling weights for a static case (top) and for a suboptimal (middle) and optimal (bottom) phase arrangements of nodes. Links contributing positively to the synchronization are yellow, and green are negative contributions. The width of the lines corresponds to the absolute value of the wave coupling weights of the links (shown next to the links) and the size of the circles corresponds to the nodes spectral capacity (dark) and strength (colored), both shown for each node.

Following these insights from synchronization in delayed networks, we define a spectral strength and capacity for each network node 


$$
C_{i}^{(f)}=\sum_{j}\left|w_{i j}^{(f)}\right|
$$

In the static case, both metrics are identical to the topological node strength, Fig. 1 (B top). The spectral strength, $S_{i}^{(f)}$, is adapted from a particle case node strength (29) by accounting for possible negative contribution of some links, i.e. negative wave coupling from an in-phase node, or a positive one from an anti-phase node, Fig. 1 (A bottom) and (B middle and bottom). The cluster $Q_{i}$ thus contains all the nodes that are in phase with the node $i$. Related to this, the spectral node capacity, $C_{i}^{(f)}$, gives the upper bound for the synchronizabillity of a given node and hence of its $S_{i}^{(f)}$, Fig. 1 (B) middle and bottom plots. Therefore it shows the strength of the node when all its links contribute positively to its dynamics. As shown in the middle and bottom plots of Fig. 1 (B), different phase arrangements of the nodes change their spectral strength, so a hub in one arrangement can become more peripheral in other arrangements, while still being constrained by their spectral capacity given by the spatio-temporal structure. In the same way, the spectral capacity of each node does not change for a given frequency, as different phase arrangements are realized.

Implications for the spectral dependent brain activity. Network-wave couplings reflect duality in the network interactions, which can be particle or wave based. The latter is reliant on the timings of the waveforms arriving from the distant nodes and the wave coupling weights change with the frequency. In the case of the connectome this causes activation of different network cores that could support the differentiation of the brain activity for various processes and frequency bands (39-42). The time delays that are crucial for the spatiotemporal network organization, are defined by the lengths of the links for homogeneous propagation velocity, i.e. $\tau_{i j}=l_{i j} / v$, which is often used as a first approximation for the actual delays $(10,26,27)$ in absence of more realistic whole-brain information (43). The cosine term modulates the strength of the links, which can even change the sign, reverting once positive interactions to become negative depending on the frequency. This is demonstrated for structural brain networks of humans and mice, Fig. 2 (upper panel).

The human data is from 100 healthy subjects (44) with volumetric parcelations performed using the Desikan-Killiany (45) atlas with 68 cortical and 16 subcortical regions, and the mouse connectome is from the Allen Institute tracing experiments (46) yielding 540 brain regions out of which 86 are part of the isocortex. The realistic range for the conduction velocity has been reported in the range of $1-10 \mathrm{~m} / \mathrm{s}$ for humans $(21,43,47,48)$ and $0.1-1 \mathrm{~m} / \mathrm{s}$ for mice $(48)$. Here we use $3.333 \mathrm{~m} / \mathrm{s}$ and $0.3333 \mathrm{~m} / \mathrm{s}$ respectively, so that the peak of the occipital activity would be within alpha frequency band for humans (Fig. 3). For both cases natural logarithm of the number of streamlines is used as a metric of the weights.

The mean spectral strength and capacity are also shown in the bottom of Fig. 2 for three different frequencies, where the phase arrangement of the nodes is obtained from an algorithm that maximizes the overall spectral strength of the network (see SI for more details). Unless specified otherwise, all the results below for the spectral strength refer to a phase arrangement that maximizes it over the network. As an example, the two graph theoretical metrices in Fig. 2 illustrate that for most of the occipital regions, the capacity at $20 \mathrm{~Hz}$ is smaller than at $10 \mathrm{~Hz}$ or $40 \mathrm{~Hz}$, whilst the spectral strength for the same regions generally decreases with increasing the frequency. 

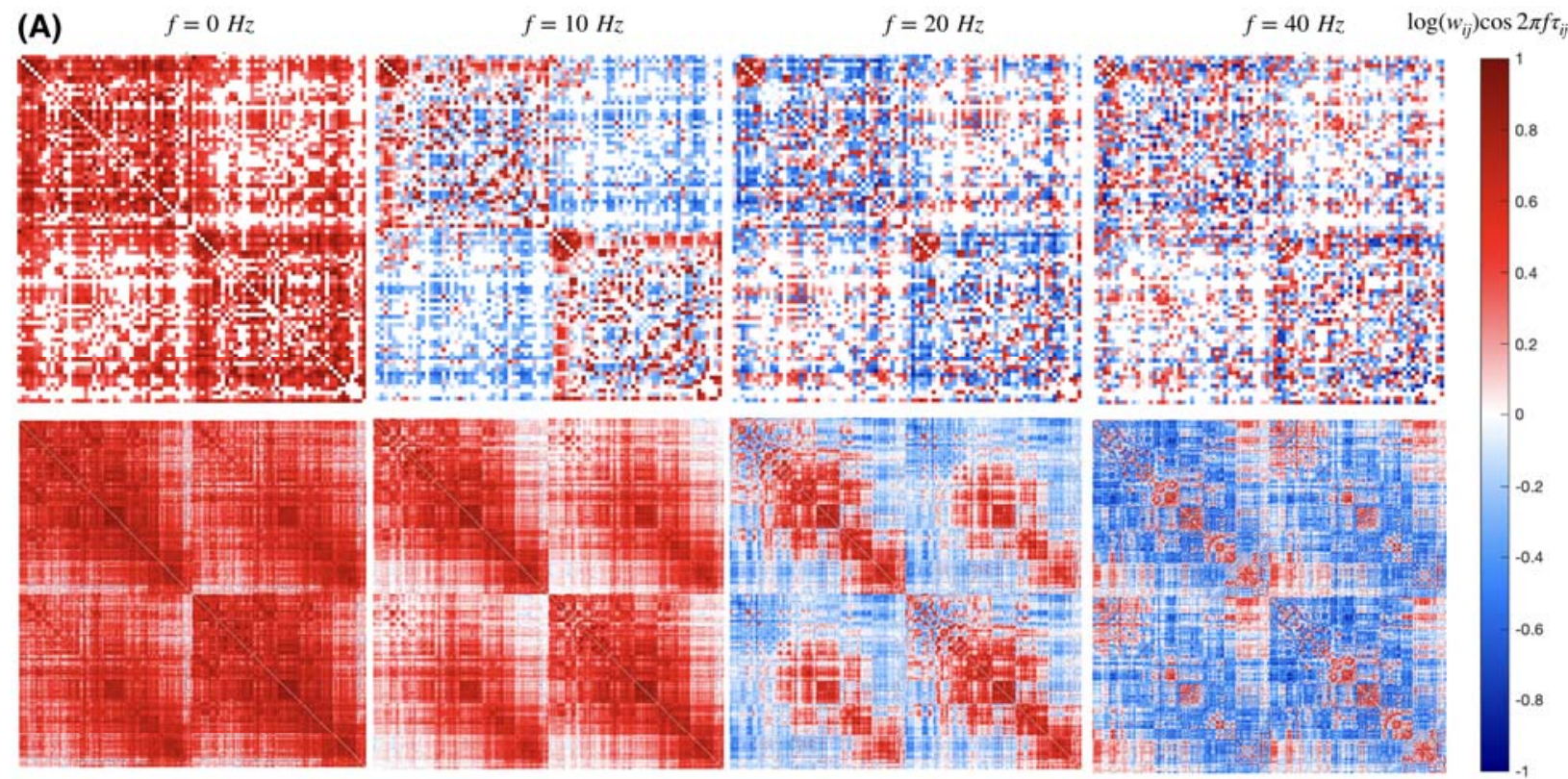

(B)
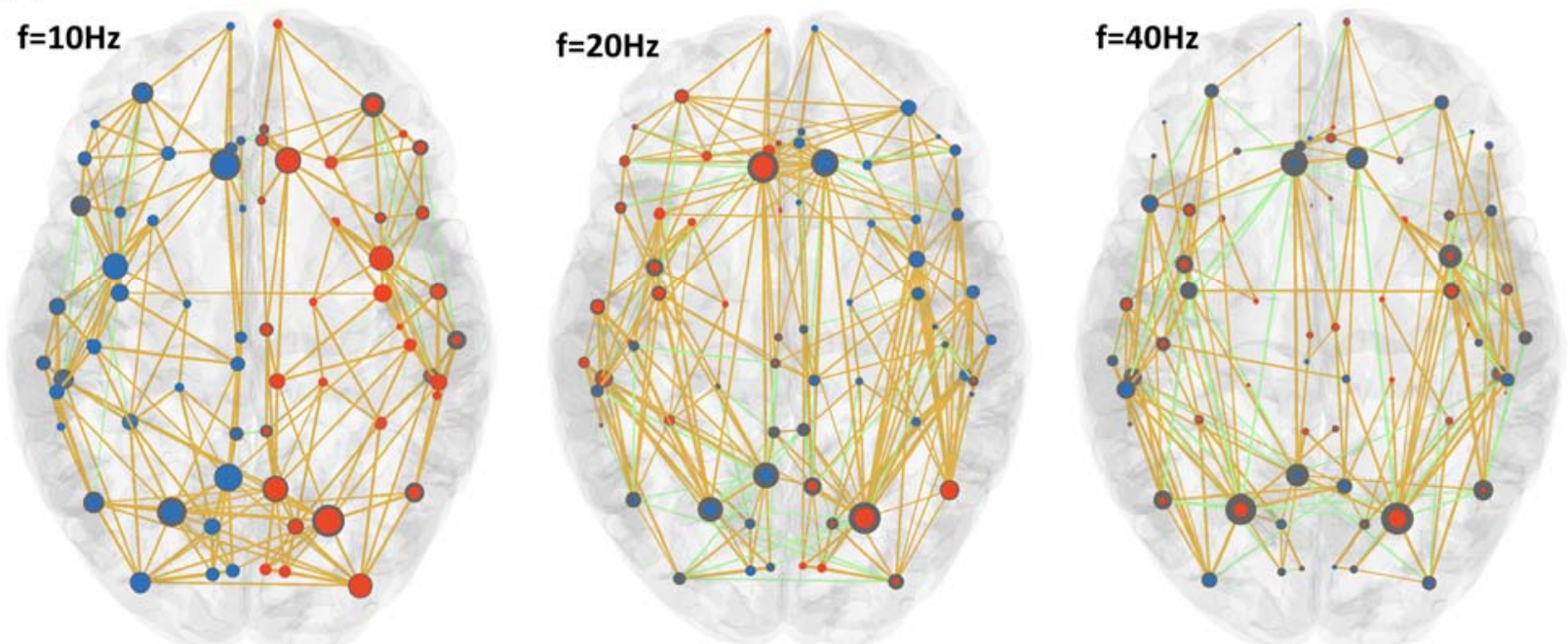

Fig. 2. Network wave couplings for human and mouse connectome, and spectral strength at different frequencies. Upper panel shows the matrices of for a human (top) and mouse (bottom) connectomes. Lower panel shows spectral strength (blue or red, indicating anti-phase arrangements) and capacity (grey) for the cortical regions of a human brain at different frequencies. The links with positive impact are yellow, and others are blue (c.f. Fig. 1 (B)).

The spatially dependent spectral affinity of the brain regions is better illustrated in Fig. 3, where the activity of different brain regions is normalized per frequency and per region. The cortical activation patterns show the regions with stronger activity than the overall mean.

Studies of functional magnetic resonance imaging (fMRI) have demonstrated that in the absence of an apparent task, fluctuations in the blood oxygenation-level dependent (BOLD) fMRI signals correlate across brain regions. These are otherwise functionally related regions in different task conditions, hence the notion of resting state networks (RSNs) (49). Such networks have been 
regularly identified across subjects using spatial independent component analysis. The most consistent network representation has been obtained for six of them (50): default mode network (DMN), visual, sensory/motor (SensMot), auditory, executive control (ExecCont) and frontalparietal (FronPar) network. We checked if any of the RSNs or lobes subnetworks have more preferred frequency range of occurrence, based on their spatiotemporal structure encompassed by the wave network coupling. For this, the spectral activity is projected across different RSNs and lobes, and from RSNs and lobes into frequency bands (Fig. 3). Interestingly, some of the most remarkable activation patterns, such as alpha activity in visual/occipital regions and the beta peak of DMN and frontal regions, are in agreement with the strongest empirically observed phenomena (51).
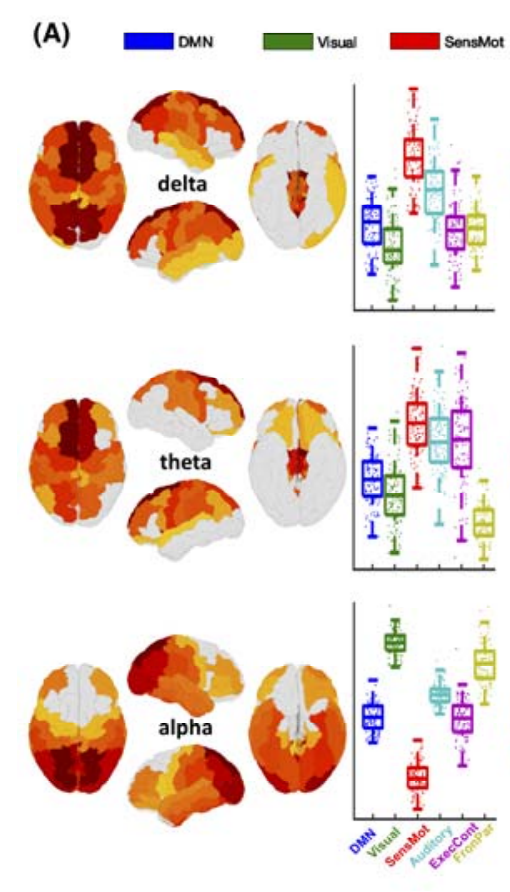
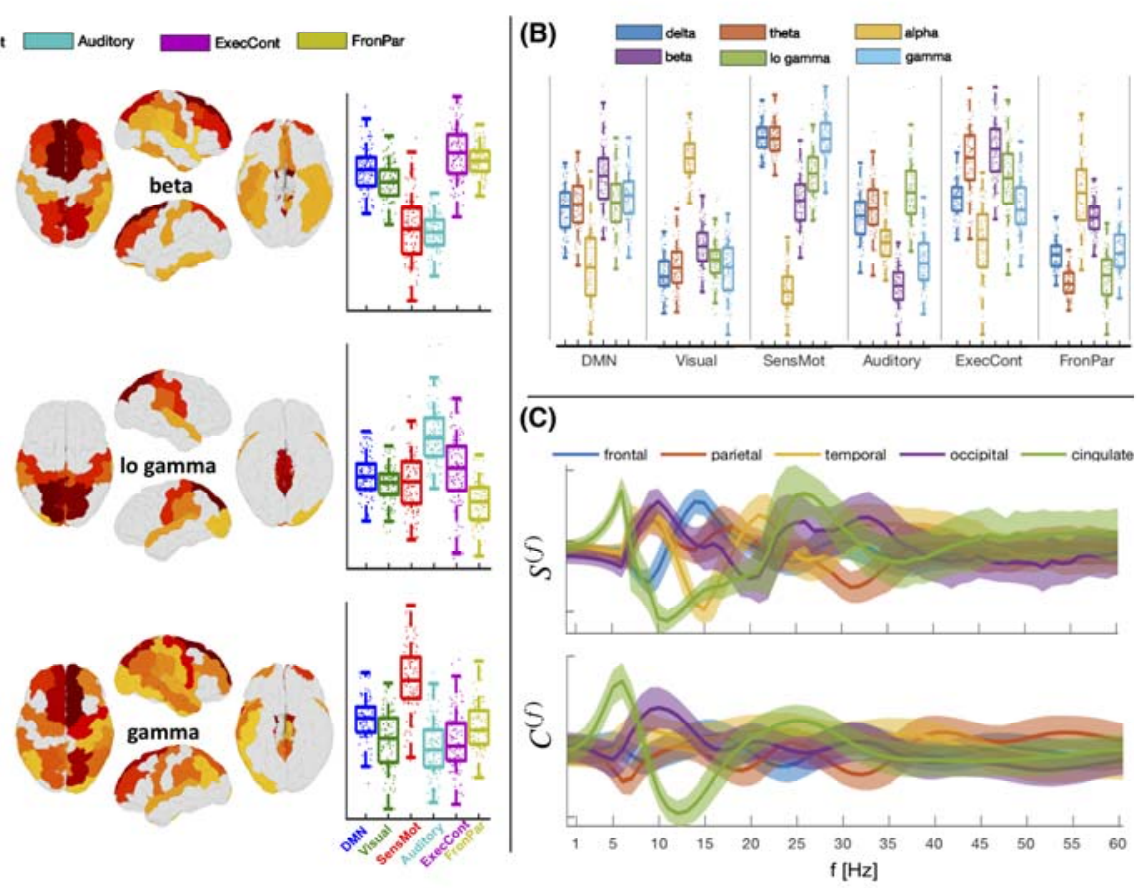

Fig. 3. Mean cortical activation patterns over 100 subjects for different frequencies. (A) Spectral strength is shown for different cortical regions and frequency bands, and activation of RSNs is projected in the frequencies. (B) Relative contribution of the frequency bands per RSN. Boxplots contain the scatter plots of all 100 subjects. (C) Mean spectral strength and capacity (lines) and regions of two standard deviations (shaded) calculated in step of $1 \mathrm{~Hz}$ for each lobe. The values per given RSN and lobe are normalized by the number of regions in that subnetwork.

Numerical confirmation for forward modeling and Linear Stability Analysis (LSA). The renormalization of the connectome also allows straightforward application of LSA for the dynamics close to a critical state for a given forward model of the oscillatory activity. In this case, the eigenvectors of the largest positive eigenvalue are guaranteed to fully capture the amplitude and the sign of the activation patterns. We choose Landau Stuart oscillators (35) to build our brain network model (BNM) (25), over the same 100 human connectomes. The local dynamics from Eq. 2 is then given as , where is a complex number, and the coupling is linear additive with . All the parameters, including the frequency and the distance from the local bifurcation are identical for every oscillator, and for each frequency the latter is set to keep the system at the edge of criticality with 
two positive eigenvalues (both variables of the 2D oscillator lose stability simultaneously, see SI for more information). In this dynamical setting the observed activation pattern for the network coupled through the renormalized connectome is guaranteed to be fully captured by the values of the first eigenvector of the $\operatorname{LSA}(27,52)$. Note that the signs of the eigenvectors correspond to the in-/anti- phase arrangements of the nodes. For consistency, in the simulated powers at different regions, the signs are also assigned as assigned by the empirical in-/anti- phase arrangements of the simulated data. Similarly, for consistent comparison between the metrices, the phase arrangements either from the data, or from the connectome, or from the eigenvalues, are explicitly assigned spectral strengths and capacities.

With the generative model. we confirm that these spectral patterns of activity are predicted by the graph theoretical metrics on the renormalized connectome, i.e. the spectral strength and capacity. The former depends on the actual phase arrangement of the oscillators. For this besides the arrangement that maximizes the overall $S^{(f)}$ (that is based only on the renormalized connectome) we also use the signs of the eigenvectors to inform the spectral strength metric about the in/anti phase organization for the specific model. In Fig. 4 (A), we show the correlation of the leading eigenvectors with spectral strength, calculated using both arrangements, for all 100 subjects. The arrangement implied by the signs of the eigenvectors takes into account the specificity of the model, and hence this case better predicts the activation of a given node. This means that the network is not always arranged in such a manner to yield maximal possible synchronization. The patterns are similar across subjects, and for frequencies larger than $40 \mathrm{~Hz}$, there seem to be specific frequencies for different subjects, for which the arrangement of the signs of the eigenvectors is quite different from those maximizing $S^{(f)}$. Spectral capacity on the other hand, has generally lower predictability than $S_{c o n n}^{(f)}$, but it is never too low or insignificant. As shown in the third column of the panel (A), all three spectral metrices exhibit much better correlation than the null metric, which is constructed assuming particle-like interactions at $\mathrm{OHz}$. Thus, only the weights of the links are considered, giving the in-strength of the nodes $C_{i}^{(0)}=$ $\sum_{j} w_{i j}$. Since the null model cannot yield anti-phase nodes, for more conservative comparison we assign it signs predicted by the arrangements of the nodes with which it is being compared.

In Fig. 4 (B) the predictability of the spectral metrices is tested by comparing them with the power obtained by simulating the model built with the same oscillators but over the full connectome, containing both weights and delays, Eq. (2). Moreover, this is extended for the subcritical and super-critical regimes of the system, along with noise. The comparison shows that each of the wave interactions metrics has consistently higher predictability than its particle-like counterpart across all the frequencies. This holds even for noisy systems far from the criticality, where the correlation is generally larger than 0.5 if the phase arrangements are taken from the data. It is worth noting that for the full system the spectral capacity seems to be more informative than the spectral strength, because the dynamics often becomes non-stationary and multi-stable due to the explicit time-delays (23). Nodes might switch from in- to anti-phase and back, and different phase arrangements lead to different spectral strengths over time. As a result, each node's average relative strength within the network might be better captured by its capacity, than by any given realization of the phase arrangements that has occurred at some point, even if that is the most dominant one. This is especially pronounced when noise is added to the system, making the spectral capacity more informative for the relative activity of the nodes almost for all the frequencies. 

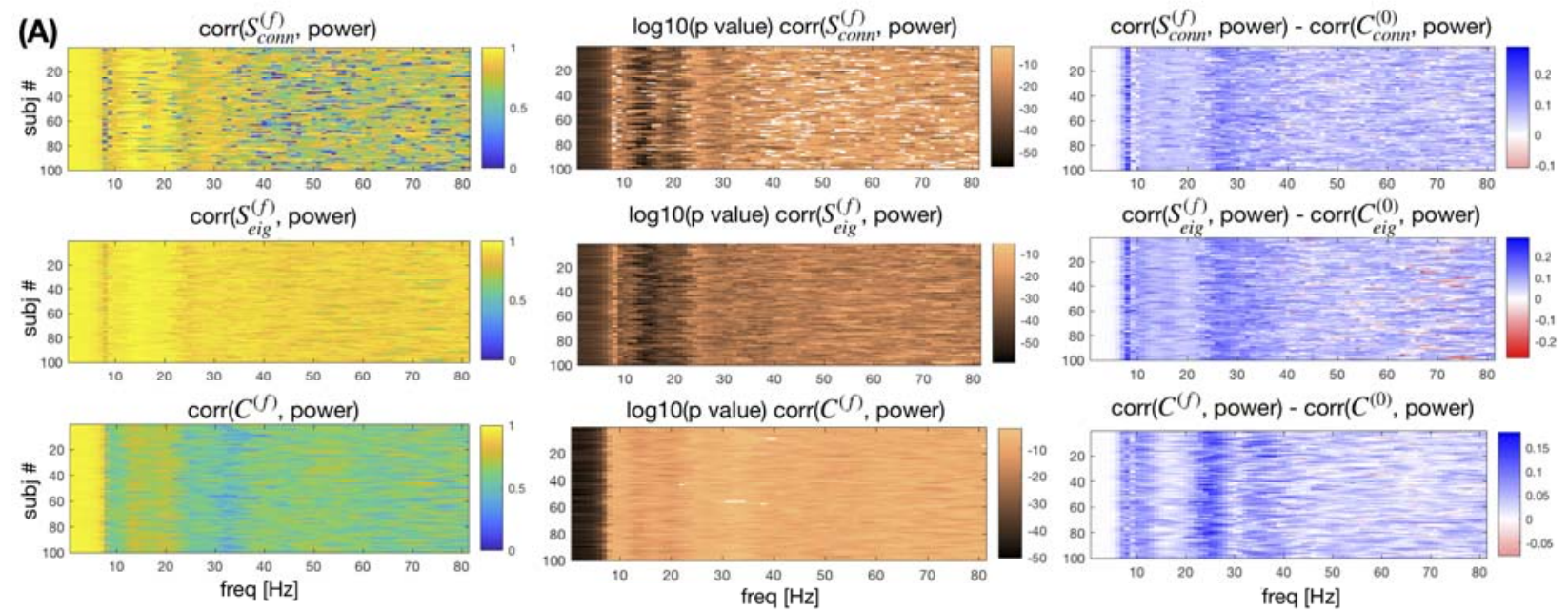

$\log 10$ (p value) $\operatorname{corr}\left(S_{\text {eig }}^{(f)}\right.$, power)
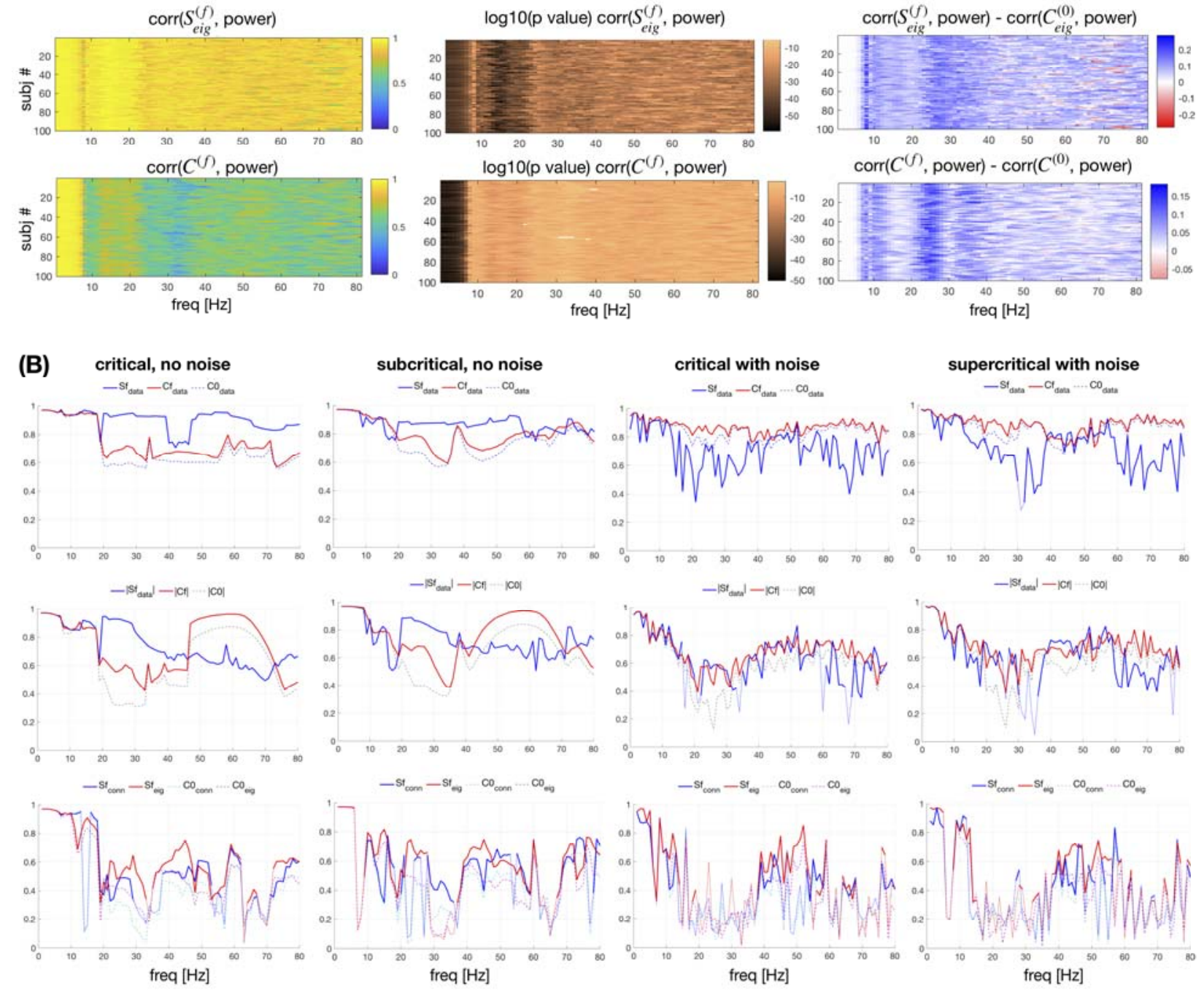

Fig. 4. Comparison with forward models based on the renormalized and the full

connectome. The power across regions from the forward model is compared with the spectral capacity and strength, where the latter is calculated either using the signs of the eigenvectors or using arrangements that maximize its value, and with the null metrics assuming particle-like interactions. The power is calculated (A) using LSA of the brain network model at criticality, constructed over the renormalized connectome or (B) with numerical simulations performed for the full connectome, with the BNM being, sub- and super-critical, and at the criticality, with and without noise. (B) For the full simulations, the most prominent phase arrangement of the simulated data is also used to set the in-/anti-phase arrangements. Correlations of the powers across frequencies (A) for all 100 subjects, as well as the p values, and the difference with the 
correlations assuming particle-like interactions, and (B) for one subject. Correlations with $\mathrm{p}<$ 0.01 are transparent in the first 2 columns of $(A)$, and thinner lines for $(B)$.

Conclusion. Wave metrics offer a new perspective and tools for characterizing dynamic networks. They integrate the wave component of information processing, which was missing in network metrics that were based solely on the connection topology (2). The incorporation of signal transmission delays in the connectome's metrics completes the characterization of the spatiotemporal skeleton, within which oscillatory brain activity can be amplified by the properties of the medium supporting it, i.e. it provides a corpus resonantiae. We have demonstrated here that the brain connectome has such properties and allows for selective and frequency dependent information processing. Notably, the wave perspective naturally leads to the formation of overlapping, albeit functionally independent, subnetworks, which cannot be derived within the particle picture. Functional independence is achieved by frequency separation of the information transmission channels. The implementation of selective communication through selective coherence has been previously hypothesized by various authors for select cognitive (53) and sensorimotor paradigms (54) and generalized to Communication Through Coherence (CTC) (32), however with no explanation to the origin of the selection of frequency bands or the spatial organization of the involved neuronal populations. The wave-particle picture of connectomics completes this view mechanistically. We propose that the activation of certain parts of the brain during different tasks can be explained as being prewired in the anatomy. It is the frequency dependent renormalized links that then facilitate CTC, making the visual network areas to be more prominent at alpha frequencies, as contrary to the sensory motor and auditory at gamma, whilst DMN is more prominent at beta frequency band, associated with the idleness of the cognitive and motor setup.

\section{Acknowledgment}

This research was supported by the EBRAINS research infrastructure, funded from the European Union's Horizon 2020 Framework Programme for Research and Innovation under the Specific Grant Agreement No. 945539 (Human Brain Project SGA3).

\section{References:}

1. S. Maslov, K. Sneppen, Specificity and stability in topology of protein networks. Science (80-. ). 296, 910-913 (2002).

2. D. S. Bassett, O. Sporns, Network neuroscience. Nat. Neurosci. 20, 353-364 (2017).

3. A. Mislove, M. Marcon, K. P. Gummadi, P. Druschel, B. Bhattacharjee, in Proceedings of the ACM SIGCOMM Internet Measurement Conference, IMC (2007).

4. E. Bullmore, O. Sporns, The economy of brain network organization. Nat. Rev. Neurosci. 13, 336-349 (2012).

5. R. Albert, A. L. Barabasi, Statistical mechanics of complex networks. Rev. Mod. Phys. 74, 47-97 (2002).

6. H. Markram et al., Reconstruction and Simulation of Neocortical Microcircuitry. Cell. 163, 456-492 (2015). 
7. O. Sporns, G. Tononi, R. Kötter, The human connectome: A structural description of the human brain. PLoS Comput. Biol. 1, 0245-0251 (2005).

8. K. J. Friston, Functional and effective connectivity: a review. Brain Connect. 1, 13-36 (2011).

9. R. Kötter, Online retrieval, processing, and visualization of primate connectivity data from the CoCoMac database. Neuroinformatics (2004), doi:10.1385/NI:2:2:127.

10. P. Sanz-Leon, S. A. Knock, A. Spiegler, V. K. Jirsa, Mathematical framework for largescale brain network modeling in The Virtual Brain. Neuroimage. 111, 385-430 (2015).

11. F. Melozzi et al., Individual structural features constrain the mouse functional connectome. Proc. Natl. Acad. Sci. U. S. A. 116, 26961-26969 (2019).

12. A. Brovelli et al., Beta oscillations in a large-scale sensorimotor cortical network: Directional influences revealed by Granger causality. Proc. Natl. Acad. Sci. 101, 98499854 (2004).

13. V. K. Jirsa et al., The Virtual Epileptic Patient: Individualized whole-brain models of epilepsy spread. Neuroimage. 145, 377-388 (2017).

14. T. Proix, F. Bartolomei, M. Guye, V. K. Jirsa, Individual brain structure and modelling predict seizure propagation. Brain. 140, 641-654 (2017).

15. C. E. Shannon, A Mathematical Theory of Communication. Bell Syst. Tech. J. (1948), doi:10.1002/j.1538-7305.1948.tb01338.x.

16. T. Schreiber, Measuring information transfer. Phys. Rev. Lett. 85, 461-464 (2000).

17. C. E. Shannon, W. Weaver, The mathematical theory of communication (Urbana, IL (1949).

18. M. Newman, Networks: An Introduction (2010).

19. G. Buzsáki, Rhythms of the Brain (2009).

20. F. Bartolomei, P. Chauvel, F. Wendling, Epileptogenicity of brain structures in human temporal lobe epilepsy: A quantified study from intracerebral EEG. Brain. 131, 18181830 (2008).

21. P. L. Nunez, R. Srinivasan, Electric fields of the brain $\square$ : the neurophysics of EEG (Oxford University Press, 2006).

22. G. Buzsáki, D. Andreas, A. Draguhn, Neuronal Oscillations in Cortical Networks. Science (80-. ). 304, 1926 (2004).

23. S. Petkoski et al., Heterogeneity of time delays determines synchronization of coupled oscillators. Phys. Rev. E - Stat. Nonlinear, Soft Matter Phys. 94, 1-7 (2016).

24. S. Petkoski, J. M. Palva, V. K. Jirsa, Phase-lags in large scale brain synchronization: Methodological considerations and in-silico analysis. PLoS Comput. Biol. 14, 1-30 (2018).

25. S. Petkoski, V. K. Jirsa, Transmission time delays organize the brain network synchronization. Philos. Trans. R. Soc. A Math. Phys. Eng. Sci. 377, 20180132 (2019). 
26. G. Deco, V. K. Jirsa, A. R. McIntosh, Emerging concepts for the dynamical organization of resting-state activity in the brain. Nat. Rev. Neurosci. 12, 43-56 (2011).

27. A. Ghosh, Y. Rho, A. R. McIntosh, R. Kötter, V. K. Jirsa, Noise during rest enables the exploration of the brain's dynamic repertoire. PLoS Comput. Biol. 4 (2008), doi:10.1371/journal.pcbi.1000196.

28. D. S. Bassett, P. Zurn, J. I. Gold, On the nature and use of models in network neuroscience. Nat. Rev. Neurosci. (2018), pp. 1-13.

29. M. Rubinov, O. Sporns, Complex network measures of brain connectivity: Uses and interpretations. Neuroimage. 52, 1059-1069 (2010).

30. J. A. Roberts et al., The contribution of geometry to the human connectome. Neuroimage. 124, 379-393 (2016).

31. A. Pikovsky, M. Rosenblum, J. (Ju $\square$ rgen) Kurths, Synchronization $\square$ : a universal concept in nonlinear sciences (Cambridge University Press, 2001; https://books.google.fr/books/about/Synchronization.html?id=FuIv845q3QUC\&redir_esc $=\mathrm{y})$.

32. P. Fries, Rhythms for Cognition: Communication through Coherence. Neuron. 88, 220235 (2015).

33. S. Palva, J. M. Palva, Discovering oscillatory interaction networks with M/EEG: Challenges and breakthroughs. Trends Cogn. Sci. 16, 219-229 (2012).

34. A. Palmigiano, T. Geisel, F. Wolf, D. Battaglia, Flexible information routing by transient synchrony. Nat. Neurosci. 20, 1014-1022 (2017).

35. Y. Kuramoto, Chemical Oscillations, Waves, and Turbulence (Springer Berlin Heidelberg, Heidelberg, 1984; http://link.springer.com/10.1007/978-3-642-69689-3), vol. 19 of Springer Series in Synergetics.

36. S. H. (Steven H. Strogatz, SYNC $\square$ : how order emerges from chaos in the universe, nature, and daily life (Theia, 2003).

37. F. A. Rodrigues, T. K. D. M. Peron, P. Ji, J. Kurths, The Kuramoto model in complex networks. Phys. Rep. 610, 1-98 (2016).

38. E. Izhikevich, Phase models with explicit time delays. Phys. Rev. E. 58, 905-908 (1998).

39. A. M. Bastos et al., Visual areas exert feedforward and feedback influences through distinct frequency channels. Neuron. 85, 390-401 (2015).

40. A. T. L. Leong et al., Long-range projections coordinate distributed brain-wide neural activity with a specific spatiotemporal profile. Proc. Natl. Acad. Sci., 201616361 (2016).

41. J. F. Mejias, J. D. Murray, H. Kennedy, X. J. Wang, Feedforward and feedback frequency-dependent interactions in a large-scale laminar network of the primate cortex. Sci. Adv. 2, e1601335 (2016).

42. J. Liu et al., Frequency-selective control of cortical and subcortical networks by central thalamus. Elife. 4, e09215 (2015).

43. L. Trebaul et al., Probabilistic functional tractography of the human cortex revisited. 
Neuroimage. 181, 414-429 (2018).

44. D. C. Van Essen et al., The WU-Minn Human Connectome Project: An overview. Neuroimage. 80, 62-79 (2013).

45. R. S. Desikan et al., An automated labeling system for subdividing the human cerebral cortex on MRI scans into gyral based regions of interest. Neuroimage. 31, 968-980 (2006).

46. S. W. Oh et al., A mesoscale connectome of the mouse brain. Nature. 508, 207-214 (2014).

47. R. Caminiti et al., Diameter, Length, Speed, and Conduction Delay of Callosal Axons in Macaque Monkeys and Humans: Comparing Data from Histology and Magnetic Resonance Imaging Diffusion Tractography. J. Neurosci. 33, 14501-14511 (2013).

48. H. Waxman, Axonal conduction delays. Scholarpedia. 7, 1451 (2012).

49. J. S. Damoiseaux et al., Consistent resting-state networks across healthy subjects. Proc. Natl. Acad. Sci. U. S. A. 103, 13848-13853 (2006).

50. M. N. Moussa, M. R. Steen, P. J. Laurienti, S. Hayasaka, Consistency of Network Modules in Resting-State fMRI Connectome Data. PLoS One. 7 (2012), doi:10.1371/journal.pone.0044428.

51. D. Mantini et al., Electrophysiological signatures of resting state networks in the human brain. Proc Natl Acad Sci. 104, 13170-13175 (2007).

52. S. Olmi, S. Petkoski, M. Guye, F. Bartolomei, V. Jirsa, Controlling seizure propagation in large-scale brain networks. PLoS Comput. Biol. 15, e1006805 (2019).

53. T. Womelsdorf et al., Modulation of neuronal interactions through neuronal synchronization. Science. 316, 1609-12 (2007).

54. J. M. Schoffelen, J. Poort, R. Oostenveld, P. Fries, Selective movement preparation is subserved by selective increases in corticomuscular gamma-band coherence. J. Neurosci. 31, 6750-6758 (2011). 